\title{
Effect of laser temperature control on Brillouin optical correlation-domain reflectometry
}

Yosuke Mizuno $^{1,2^{*}}$, Naoki Motoishi ${ }^{2}$, Kohei Noda $^{2}$, Heeyoung Lee $^{3}$, and Kentaro Nakamura ${ }^{2}$ 1 Faculty of Engineering, Yokohama National University, Yokohama 240-8501, Japan

2 Institute of Innovative Research, Tokyo Institute of Technology, Yokohama 226-8503, Japan

3 College of Engineering, Shibaura Institute of Technology, Tokyo 135-8548, Japan

*E-mail: mizuno-yosuke-rg@ynu.ac.jp

\begin{abstract}
We show that $1-{ }^{\circ} \mathrm{C}$ drift of laser temperature yields a strain error of $\sim 8 \mu \varepsilon$ in Brillouin optical correlation-domain reflectometry. Under the assumption that the strain measurement accuracy is $+/-20 \mu \varepsilon$ (severe requirement), $+/-2.5-{ }^{\circ} \mathrm{C}$ drift of the laser temperature can be permitted for proper system operation. We prove this prediction by performing distributed strain measurements at different laser temperatures. Our results indicate that, if we allow a strain measurement accuracy of approximately $+/-100 \mu \varepsilon$ (or more), we can even remove the thermal control of the laser, leading to cost reduction and downsizing of the system.
\end{abstract}

Distributed strain and temperature sensors are key tools for monitoring the conditions of various civil infrastructures. In particular, distributed sensing techniques based on Brillouin scattering in optical fibers have been the focus of attention because of their ability to detect sub-centimeter strained or heated sections along long optical fibers. ${ }^{1)}$ Based on the configurations of light injection to sensing fibers, Brillouin sensors can be divided into twoend-access "analysis" and one-end-access "reflectometry". The former includes Brillouin optical time-, frequency-, and correlation-domain analysis (BOTDA, ${ }^{2-5)}$ BOFDA, ${ }^{6-8)}$ and BOCDA), ${ }^{9-13)}$ whereas the latter includes Brillouin optical time-, frequency-, and correlationdomain reflectometry (BOTDR, ${ }^{14-16)}$ BOFDR,${ }^{17)}$ and BOCDR). ${ }^{18-25)}$ In general, analysis systems exhibit higher signal-to-noise ratios (SNRs) due to the use of stimulated Brillouin scattering. However, despite their lower SNRs, reflectometers based on spontaneous Brillouin scattering have higher degrees of freedom in embedding the sensing fibers into structures; in addition, measurements can be continued even when the sensing fibers are broken. Needless to say, each reflectometry has its own merits and demerits, and here, we focus on BOCDR. 
BOCDR was first proposed in 2008 and has been extensively studied since then. ${ }^{18)}$ Its performance has been significantly improved; ${ }^{19-25)}$ for instance, the trade-off relation between the spatial resolution and the measurement range has been mitigated by temporal-gate scheme and double-modulation scheme. ${ }^{20,21)}$ Plastic-fiber-based operation has also been demonstrated. ${ }^{22)}$ In addition, one of the most recent achievements is real-time operation of the system by phase-detected BOCDR and slope-assisted BOCDR. 23,24) However, implementation cost of BOCDR is not yet sufficiently low, though some trials have been made. ${ }^{25)}$ Thus, it is extremely important to reduce the cost of the system for practical use in future.

In most of the laser-based sensing systems with high accuracy, the optical wavelength is stabilized by precise thermal control of the laser, which is often performed using thermoelectric cooling (TEC) based on the Peltier effect. ${ }^{26,27)}$ If the thermal control is absent in the laser, the output wavelength significantly fluctuates, leading to measurement error. As Brillouin frequency shift (BFS) is in inverse proportion to wavelength, ${ }^{1)}$ Brillouin sensors are also subject to error. However, as all the configurations of BOCDR are based on interferometry, ${ }^{18-25)}$ the influence of the wavelength fluctuations on the measurement accuracy can be suppressed to a certain range. If thermal control of the laser turns out unnecessary (at least for some applications that do not require high accuracy), the implementation cost of BOCDR will be reduced.

In this work, first, we investigate the thermal behavior of a semiconductor laser and its influence on the BFS measured by heterodyne detection. Then, we discuss the relation between the accuracy of the thermal control of the laser and the measurement accuracy of strain and temperature. Finally, we experimentally show that, if ambient temperature of the sensing fiber is constant and a moderate accuracy is acceptable, a TEC-free laser can be used for distributed strain measurement based on BOCDR for cost reduction.

BOCDR is a distributed sensing technique with operation by single-end light injection, relatively high spatial resolution, random accessibility to sensing points, and a high sampling rate. ${ }^{18-25)}$ It operates based on the synthesis of an optical coherence function (SOCF). ${ }^{28)}$ The optical output from a laser is divided into pump and reference lights. The pump light is injected into a sensing fiber, and the Stokes light is directed into a photodetector. The reference light is used as an optical local oscillator. The electrical beat signal of the two light beams is monitored using an electrical spectrum analyzer (ESA). Because of the frequency difference of $\sim 11 \mathrm{GHz}$ between the Stokes and reference lights, ${ }^{1)}$ this configuration is called self-heterodyne. 
To resolve the position along the sensing fiber, the optical frequency of the laser output is often modulated in a sinusoidal waveform by directly modulating the injection current of the laser. From the viewpoint of temporal averaging, the coherence function is synthesized into a series of periodical peaks, whose interval is inversely proportional to the frequency of the sinusoidal modulation. We control the modulation frequency to generate only one correlation peak within the range of the sensing fiber, so that only the Brillouin-scattered light at that peak has high correlation with the reference light, giving high heterodyne output. The peak frequency observed using the ESA gives the BFS caused at the specific position. By sweeping the modulation frequency, the position of the correlation peak is scanned along the sensing fiber, and thus the distribution of the Brillouin gain spectrum (BGS) or the BFS can be obtained. The spatial resolution and the measurement range of standard BOCDR are expressed as Eqs. (15) and (16) in Ref. 19, respectively.

In general, if TEC is not used in a laser, its output wavelength fluctuates. Therefore, if a TEC-free laser is employed in BOCDR, a certain amount of error in strain and temperature measurements is anticipated, because the BFS is inversely proportional to wavelength. ${ }^{1)}$ It is thus of considerable interest to quantitatively evaluate the influence of the wavelength fluctuations on the measurement accuracy of BOCDR and to discuss under what conditions such a TEC-free laser can be used in BOCDR for cost reduction.

As preliminary experiments, we investigated the basic characteristics of a typical commercially-available semiconductor laser (NX8563LB, NEC; rated maximum temperature $=65^{\circ} \mathrm{C}$; spectral linewidth $=\sim 1 \mathrm{MHz}$ ) connected to a temperature controller (LDC-3900, ILX Lightwave; displayed temperature stability $<0.01^{\circ} \mathrm{C}$ ). First, when TEC was active, the output frequency was measured as a function of a laser temperature at three different driving currents (Fig. 1(a)). Note that BOCDR has been driven around these driving currents. The frequency decreased with increasing temperature and current. Next, at three different driving currents, the temporal variations of the laser temperature were measured (Fig. 1(b); at time $t=0$, TEC was switched off; the initial temperature was set to room temperature $\left.\left(26.1^{\circ} \mathrm{C}\right)\right)$. Regardless of the current, the laser temperature increased with time and became almost constant at $t=$ $\sim 60 \mathrm{~s}$. Then, the saturated laser temperature (measured at $t=120 \mathrm{~s}$ ) was plotted as a function of the driving current (Fig. 1(c)). The saturated temperature increased with increasing driving current, following a quadratic fit reflecting Joule's first law. Seeing that the saturated temperature even at $100 \mathrm{~mA}$ is much lower than the rated maximum temperature, it appears that the laser is not destroyed by excessive heat under normal operation of BOCDR even when TEC is not employed. 
The experimental setup of BOCDR used in this work is depicted in Fig. 2. The pump power was $20 \mathrm{dBm}$, and the sensing fiber was a 3.6-m-long silica single-mode fiber (SMF). A $2.0-\mathrm{km}$ delay line and a polarization scrambler were inserted in the reference path. For distributed measurements, the modulation frequency was $1.1730-1.1744 \mathrm{MHz}$ and the modulation amplitude was $2.84 \mathrm{GHz}$, corresponding to the spatial resolution of $0.29 \mathrm{~m}$ and the measurement range of $87.6 \mathrm{~m}$. The BGS observed at a single measurement position was averaged 10 times on the ESA. The BFS was simply calculated as the frequency at which the BGS became maximal. The video bandwidth and the resolution bandwidth of the ESA were $1 \mathrm{kHz}$ and $3 \mathrm{MHz}$, respectively. The total measurement time was $10 \mathrm{~s}$. The room temperature was $25.9^{\circ} \mathrm{C}$.

First, we experimentally investigated the dependence of the laser temperature on the BFS. Here, to observe the light Brillouin-scattered in the whole length of the sensing fiber to enhance the SNR, the optical frequency was not modulated. The normalized BGS dependence on the laser temperature is shown in Fig. 3(a). With increasing laser temperature, the BGS shifted to lower frequency. The BFS is then plotted as a function of the laser temperature (Fig. 3(b)). The BFS linearly decreased with increasing laser temperature with a coefficient of $0.41 \mathrm{MHz} /{ }^{\circ} \mathrm{C}$. Considering the strain and temperature dependence coefficients of the BFS at $1.55 \mu \mathrm{m}$ are $+480 \mathrm{MHz} / \%$ and $+1.18 \mathrm{MHz} /{ }^{\circ} \mathrm{C},{ }^{29,30)}$ respectively, we can estimate that $1-{ }^{\circ} \mathrm{C}$ drift of the laser temperature corresponds to strain and temperature measurement errors of $0.0008 \%(=8 \mu \varepsilon)$ and $0.34^{\circ} \mathrm{C}$. Let us suppose that the ambient temperature of the sensing fiber is constant. Then, even if we assume that the strain measurement accuracy of BOCDR is $+/-20 \mu \varepsilon$ (applicable to detection of local yielding or cracking in structure; note that the actual accuracy, which depends on various experimental conditions such as spatial resolution, pump power, signal processing (averaging, fitting, etc), and many others, is much worse than this), $+/-2.5-{ }^{\circ} \mathrm{C}$ drift of the laser temperature is permitted in the BOCDR operation.

Subsequently, to verify this result, we performed distributed strain measurements when the laser temperature was $20^{\circ} \mathrm{C}$ and $25^{\circ} \mathrm{C}$ (imitating a drift of $+/-2.5^{\circ} \mathrm{C}$ ). Strains of $0.4 \%$ and $0.6 \%$ were applied to a $1.0-\mathrm{m}$-long section (1.0-2.0 m from the proximal end) of the $3.6-\mathrm{m}$ sensing fiber. The normalized BGS distributions measured when $0.4 \%$ strain was applied at the laser temperatures of $20^{\circ} \mathrm{C}$ and $25^{\circ} \mathrm{C}$ are shown in Figs. 4(a) and 4(b), respectively. In both results, the local shift of the BGS at the strained section was clearly detected. Finally, Figs. 5(a) and 5(b) show the comparisons of the BFS distributions at the laser temperatures of $20^{\circ} \mathrm{C}$ and $25^{\circ} \mathrm{C}$, measured when $0.4 \%$ and $0.6 \%$ strains were applied, respectively. It is clear that, regardless of the amount of strain, the discrepancies caused by the two different 
laser temperatures (in average, $-0.10 \mathrm{MHz}$ at $0.4 \%$ and $+0.48 \mathrm{MHz}$ at $0.6 \%$ ) were negligibly small compared to the inherent fluctuations of the BFS (the standard deviations from the theoretical trends at $20^{\circ} \mathrm{C}$ were $+/-11.3 \mathrm{MHz}$ at $0.4 \%$ and $+/-11.8 \mathrm{MHz}$ at $0.6 \%$ ). This result indicates that, for proper operation of BOCDR, the laser temperature does not need to be precisely controlled (e.g., $<+/-0.01^{\circ} \mathrm{C}$ ) and can be roughly adjusted with an accuracy of $+/-$ $2.5^{\circ} \mathrm{C}$. Considering the result of the preliminary experiments (Fig. 1(c)), if we allow the strain measurement accuracy of approximately $+/-100 \mu \varepsilon$ or more, even removal of the thermal control of the laser will be feasible.

In conclusion, the thermal behavior of a commercial laser and its influence on the BFS were investigated, and $1-^{\circ} \mathrm{C}$ drift of the laser temperature was estimated to correspond to strain measurement error of $8 \mu \varepsilon$. This means that, assuming that the strain measurement accuracy of BOCDR is $+/-20 \mu \varepsilon$ (severe requirement), $+/-2.5-{ }^{\circ} \mathrm{C}$ drift of the laser temperature is permitted in the BOCDR operation. Then, we experimentally proved this prediction by performing distributed strain measurements at the laser temperatures of $20^{\circ} \mathrm{C}$ and $25^{\circ} \mathrm{C}$. Our results indicate that, allowing the strain measurement accuracy of approximately $+/-100 \mu \varepsilon$ (or more), we can even remove the thermal control of the laser. We believe that employment of a TEC-free laser will enable cost reduction and downsizing of the BOCDR system (note that our conclusion will hold true not only for BOCDR but also for all types of Brillouin analysis/reflectometry based on heterodyne detection), promoting its practical use in the future.

\section{Acknowledgments}

This work was supported by JSPS KAKENHI Grant Numbers 17H04930 and 17J07226. 


\section{References}

1) G. P. Agrawal, Nonlinear Fiber Optics (Academic Press, San Diego, CA, 1995).

2) T. Horiguchi and M. Tateda, J. Lightwave Technol. 7, 1170 (1989).

3) D. Zhou, Y. Dong, B. Wang, C. Pang, D. Ba, H. Zhang, Z. Lu, H. Li, and X. Bao, Light: Sci. Appl. 7, 32 (2018).

4) Y. Peled, A. Motil, L. Yaron, and M. Tur, Opt. Express 19, 19845 (2011).

5) M. A. Soto, J. A. Ramírez, and L. Thévenaz, Nat. Commun. 7, 10870 (2016).

6) D. Garus, K. Krebber, F. Schliep, and T. Gogolla, Opt. Lett. 21, 1402 (1996).

7) R. Bernini, A. Minardo, and L. Zeni, IEEE Photonics J. 4, 48 (2012).

8) D. Garus, T. Gogolla, K. Krebber, and F. Schliep, J. Lightwave Technol. 15, 654 (1997).

9) K. Hotate and T. Hasegawa, IEICE Trans. Electron. E83-C, 405 (2000).

10) K. Y. Song, M. Kishi, Z. He, and K. Hotate, Opt. Lett. 36, 2062 (2011).

11) A. López-Gil, S. Martin-Lopez, and M. Gonzalez-Herraez, Opt. Lett. 42, 3924 (2017).

12) Y. Okawa, R. K. Yamashita, M. Kishi, and K. Hotate, Opt. Express 28, 6981 (2020).

13) W. Zou, C. Jin, and J. Chen, Appl. Phys. Express 5, 082503 (2012).

14) T. Kurashima, T. Horiguchi, H. Izumita, S. Furukawa, and Y. Koyamada, IEICE Trans. Commun. E76-B, 382 (1993).

15) A. Masoudi, M. Belal, and T. P. Newson, Opt. Lett. 38, 3312 (2013).

16) G. Tu, X. Zhang, Y. Zhang, Z. Ying, and L. Lv, Electron. Lett. 50, 1624 (2014).

17) A. Minardo, R. Bernini, R. Ruiz-Lombera, J. Mirapeix, J. M. Lopez-Higuera, and L. Zeni, Opt. Express 24, 29994 (2016).

18) Y. Mizuno, W. Zou, Z. He, and K. Hotate, Opt. Express 16, 12148 (2008).

19) Y. Mizuno, W. Zou, Z. He, and K. Hotate, J. Lightwave Technol. 28, 3300 (2010).

20) Y. Mizuno, Z. He, and K. Hotate, Opt. Express 17, 9040 (2009).

21) Y. Mizuno, Z. He, and K. Hotate, Opt. Express 18, 5926 (2010).

22) N. Hayashi, Y. Mizuno, and K. Nakamura, J. Lightwave Technol. 32, 3999 (2014).

23) Y. Mizuno, N. Hayashi, H. Fukuda, K. Y. Song, and K. Nakamura, Light: Sci. Appl. 5, e16184 (2016).

24) H. Lee, N. Hayashi, Y. Mizuno, and K. Nakamura, IEEE Photonics J. 8, 6802807 (2016).

25) Y. Mizuno, G. Han, K. Noda, H. Lee, and K. Nakamura, Electron. Lett. 55, 754 (2019).

26) K. P. Pipe, R. J. Ram, and A. Shakouri, IEEE Photonics Technol. Lett. 14, 453 (2002).

27) M. Labudovic and J. Li, IEEE Trans. Comp. Packaging Technol. 27, 724 (2004).

28) K. Hotate and Z. He, J. Lightwave Technol. 24, 2541 (2006).

29) T. Horiguchi, T. Kurashima, and M. Tateda, IEEE Photonics Technol. Lett. 1, 107 (1989).

30) T. Kurashima, T. Horiguchi, and M. Tateda, Appl. Opt. 29, 2219 (1990). 


\section{Figure Captions}

Fig. 1. (Color online) (a) Output frequency vs. laser temperature at three driving currents. The solid lines are linear fits. (b) Temporal variations of the laser temperatures at three driving currents. (c) Saturated laser temperature $v s$. driving current. The dotted curve is a quadratic fit.

Fig. 2. (Color online) Experimental setup of BOCDR. EDFA: erbium-doped fiber amplifier.

Fig. 3. (Color online) (a) Normalized BGS observed at different laser temperatures. (b) BFS dependence on the laser temperature. The dotted curve is a linear fit.

Fig. 4. (Color online) Normalized BGS distributions at laser temperatures of (a) $20^{\circ} \mathrm{C}$ and (b) $25^{\circ} \mathrm{C} ; 0.4 \%$ strain was applied.

Fig. 5. (Color online) BFS distributions at laser temperatures of $20^{\circ} \mathrm{C}$ and $25^{\circ} \mathrm{C}$; strains of (a) $0.4 \%$ and (b) $0.6 \%$ were applied. 


\section{Figures}

Fig. 1.

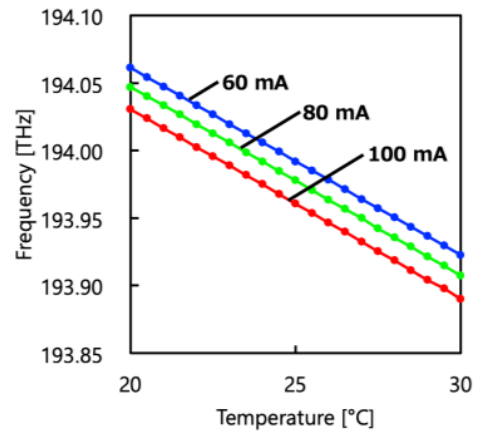

(a)

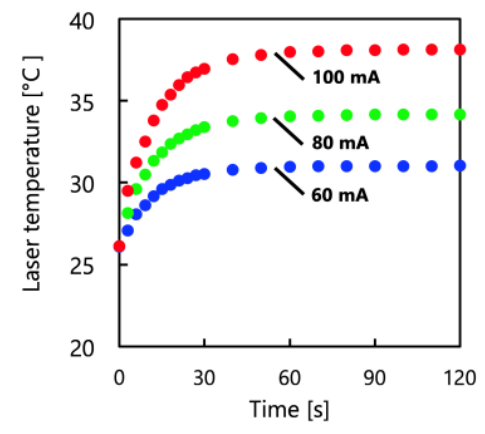

(b)

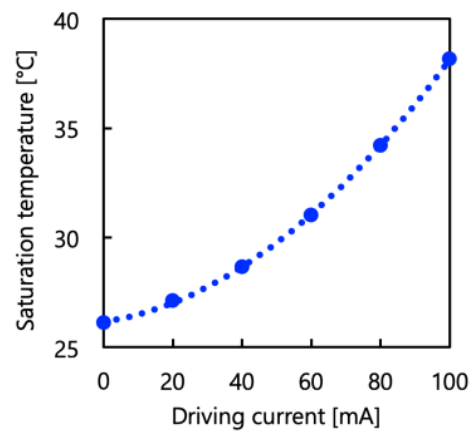

(c)

Fig. 2.

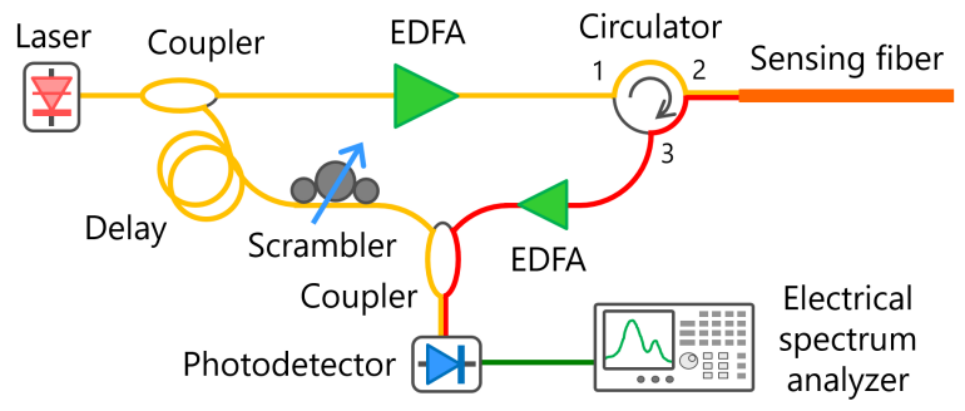

Fig. 3

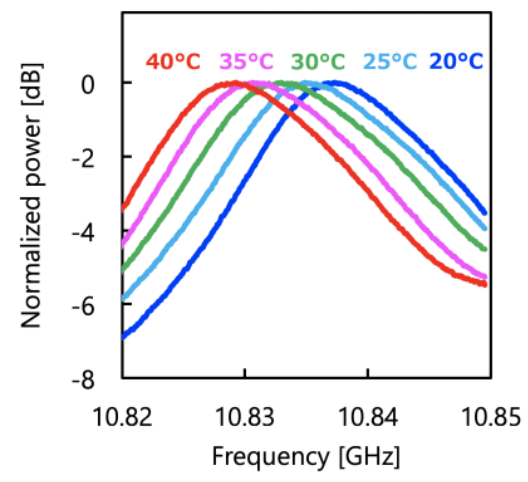

(a)

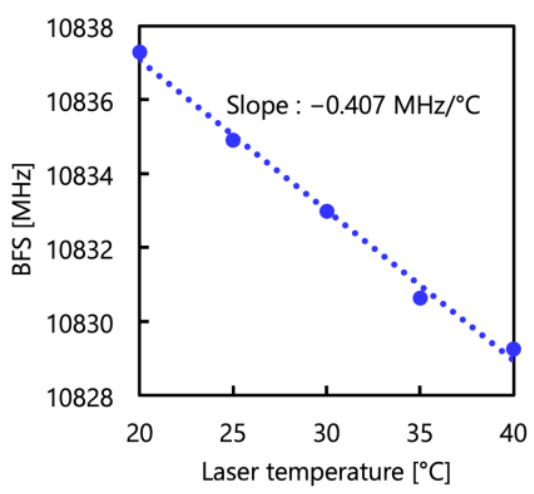

(b) 
Fig. 4

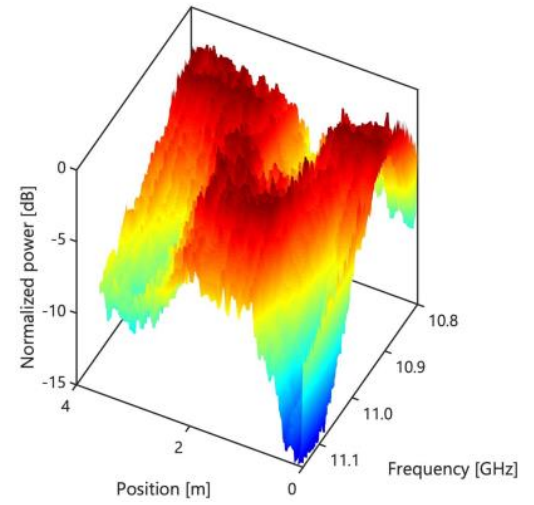

(a)

Fig. 5

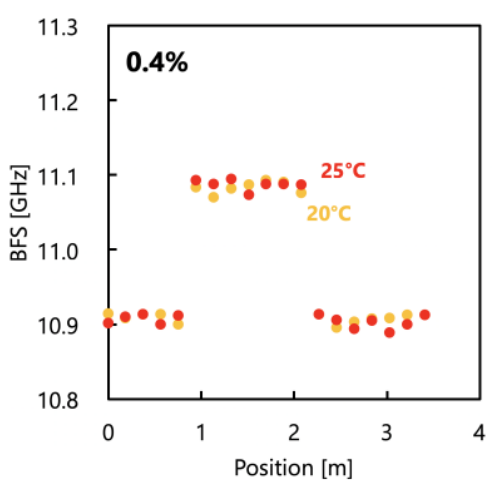

(a)

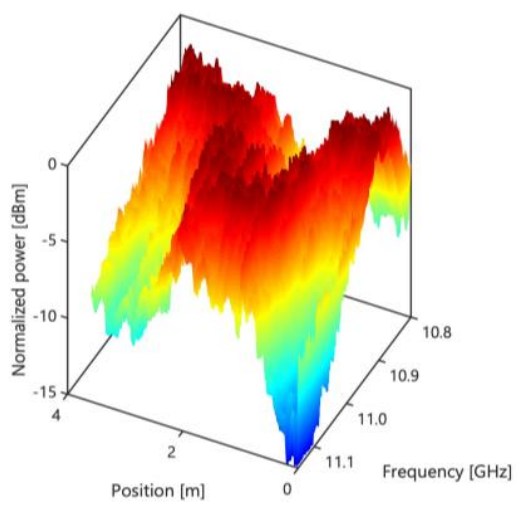

(b)

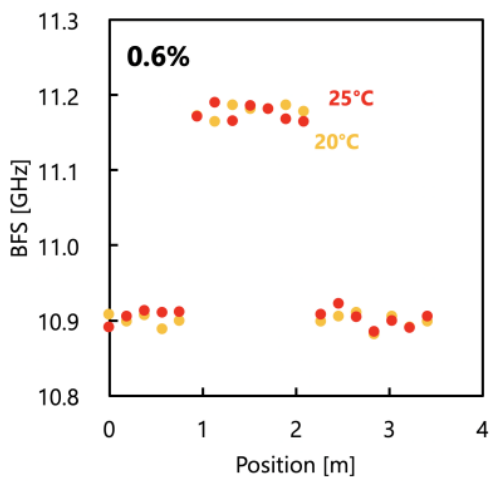

(b) 